\title{
Mathematical Modelling and Extremum Seeking Control of Cascade of Two Bioreactors for Production of Hydrogen and Methane
}

\author{
V. Lakov, E. Chorukova, I. Simeonov
}

Key Words: Extremum seeking control; cascade control; anaerobic digestion bioreactors; biogas production optimization; non-linear models.

\begin{abstract}
A mathematical model of a cascade of two continuously stirred anaerobic bioreactors is presented in this paper and the extremum seeking control is applied on it. The dilution rate of the first bioreactor is chosen as control action and the joint biogas flow rate both bioreactors is the measured output to be maximized. The concentration of inlet organics is accepted as the disturbance. The correction of the working volume of the second bioreactor is studied as the inner loop of the cascade control system. The outer control loop is represented by the extremum seeking controller, which finds the optimum control action and reacts to the disturbance. Computer simulation studies show the feasibility of this scheme.
\end{abstract}

\section{Introduction}

The human population is constantly growing and with it, the environmental pollution and energy demands are increasing. Dumping the used materials generates problems with their safe storage and simultaneously their value for modern circular world economy is lost. Keeping the raw materials longer in circulation will mitigate environmental pollution and will make our world looks better in several ways.

Anaerobic digestion of organic wastes (AD) is a relatively cheap method for realization of two tasks: depollution of organic wastes and production of energy in the form of biogas (hydrogen and methane) at the same time [1]. The outlet material (digestate) can be used for fertilization of farmlands and other territories. That is why it is important to find ways of increasing the performance of the AD process and maintain the stability of the processes $[11,12]$.

The effectiveness of the AD processes depends on many factors such as the inlet material, physical and chemical conditions in the bioreactors, bioreactor types and the microorganisms. Due to these diverse factors and the difficulties in measuring important indicators in real-time or the high price of such systems, which can be used for modelling and control of the process, it is very difficult to operate biogas installations with high performance. The extremum seeking control (ESC) does not require process model to find optimum control action, so ESC is very suitable for controlling biogas installations [2-5]. Chaining two AD biore- actors in cascade system leads to dual digestion by different microorganisms which increase transformation of organics into hydrogen and methane.

The aim of the paper is examining the possibility of using ESC controller on cascade of two bioreactors with non-linear mathematical models and the studying of overall performance of the cascade system in the presence of disturbance in the inlet organic concentration. The impact of a more complex model of the first bioreactor on system performance is also examined.

\section{Anaerobic Digestion Process}

The production of hydrogen in addition to the wide spread methane production is conditioned by gaining economic importance of the hydrogen in today's world. Burning of methane is accompanied by releasing of carbon dioxide which has significant effect on global warming. In table 1 are given the typical biogas components concentrations.

Table 1. Typical chemical composition of the biogas

\begin{tabular}{|c|c|}
\hline Gas & $\begin{array}{c}\text { Concentration, } \\
\text { \% Vol }\end{array}$ \\
\hline Methane $-\mathrm{CH}_{4}$ & $50-80$ \\
\hline Hydrogen $-\mathrm{H}_{2}$ & $5-10$ \\
\hline Carbon dioxide $-\mathrm{CO}_{2}$ & $20-50$ \\
\hline Water vapor $-\mathrm{H}_{2} \mathrm{O}$ & $2-10$ \\
\hline Nitrogen $-\mathrm{N}_{2}$ & $0-2$ \\
\hline Hydrogen sulfide $-\mathrm{H}_{2} \mathrm{~S}$ & $0-0.4$ \\
\hline
\end{tabular}

The AD processes consist of four main steps: hydrolysis, acidogenesis, acetogenesis and methanogenesis. Each of these steps is conducted by separate microorganism. These steps go simultaneously in the bioreactor and lead to formation of diverse products. The pathways for producing different products are several and can alter under the influence of conditions in the bioreactor. Transformations of organic compounds in the branches of the diagram given on figure 1 take places with different rates and efficiency, due to different microorganism involved. 
Complex organic substances entering the bioreactor can't be used directly by hydrogen and methane producing microorganisms. During first step, the hydrolysis, the long organic molecules are cut to shorter ones via enzymes secreted by bacteria. Next steps convert this products to even shorter ones (acidogenesis and acetogenesis). The last step, methanogenesis, converts acetates into methane. This gas is produced also from hydrogen and carbon dioxide released in the previous steps. Another pattern is the conversion of acetate into hydrogen and carbon dioxide and vice versa by another type of microorganisms. From the diagram on figure 1 is obvious that biogas production by $\mathrm{AD}$ of organic wastes have potential for optimization by microorganisms' selection, bioreactor conditions and processed materials.

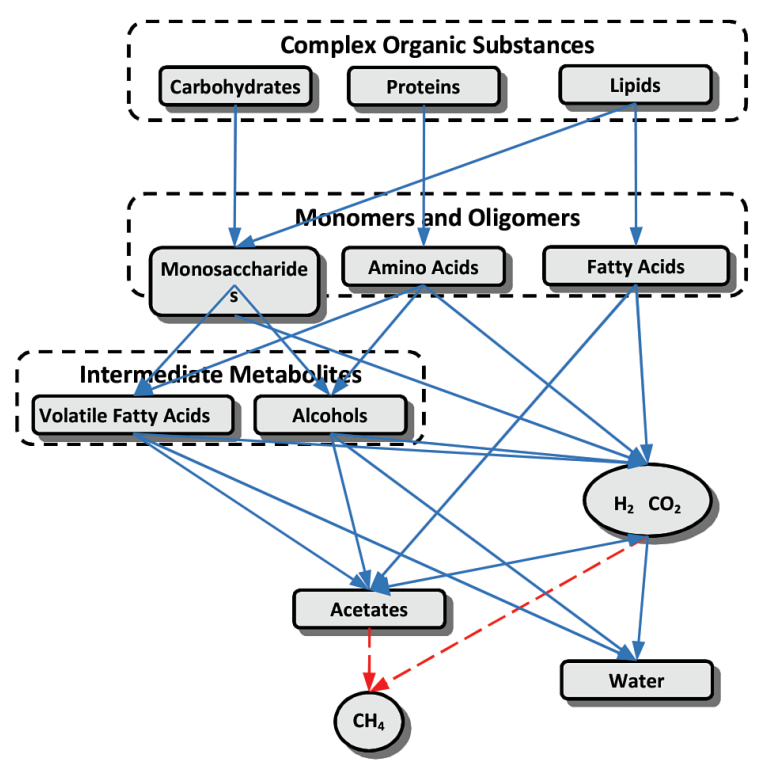

Figure 1. General diagram of the AD process

Another way for efficiency boosting is the separation of the AD steps in two different bioreactors each with optimum conditions tuned for given microorganisms [4,8]. Each of them has exceptive environmental requirements which lead to sub-optimal process performance if only one bioreactor is used. This means that the cost of the biogas will be higher and it won't be economically attractive. It is known that the application of two-phase process considerably increases biogas production. Diagram of a typical two-phase cascade system of bioreactors is given on figure 2.

The aim of such system is hydrogen production to take place only in the first bioreactor and methane production only in the second bioreactor. Organic wastes are first fed in the hydrogen bioreactor and after that they are transferred to the second bioreactor for consecutive conversion of remaining organics into methane. This scheme provides better extraction of organic pollutants than the single bioreactor scheme. This leads to more biogas produced and lowering to the greater extend the organic contents in the output product.

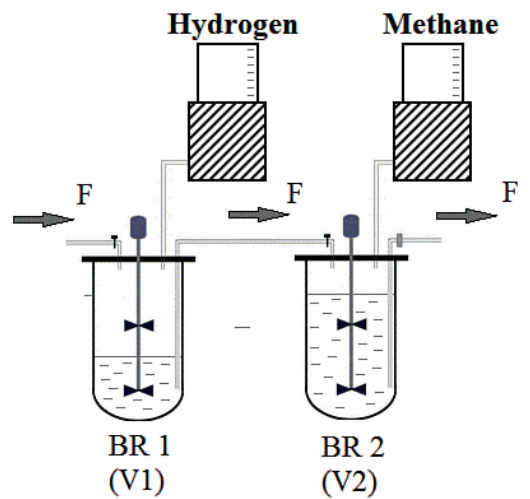

Figure 2. Diagram of a cascade of two bioreactors for separate production of hydrogen and methane

Except the selection of suitable microorganisms in the two bioreactors, it is necessary to comply with some technological constrains in order the cascade system to function in the best possible way. The specific growth rates of the microorganisms producing methane is significantly lower than that of the microorganisms in the hydrogen bioreactor. If the inlet flow of the reactor is too high, there won't have enough time for the methanogenic microorganisms to grow and they will be washed out by the output flow of the bioreactor. If the inlet flow of the first bioreactor is kept also low, this will strongly limit the hydrogen yield. The solution of this problem is to make the second bioreactor with higher volume, so the washout effect is avoided.

In order to achieve also the maximum methane yield, it is necessary to determine the how large the second bioreactor in comparison to the hydrogen one must be. The ratio of the working volumes, have to be such that the methane bioreactor works in its optimal working point when the hydrogen bioreactor is in its optimum point. The inner control loop of the cascade system is represented by the working volume of the second bioreactor. The determination of the optimum dilution rate of the inlet organic substrate of the first bioreactor is done by ESC controller.

\section{Extremum Seeking Control}

The ESC is a type of optimal control, which is applied in situations where plant model and/or objective function are not completely known, but input-output signals are measurable and this function has an extremum. The aim of this approach is through constant change of the control input to search for such value, that the objective function is maximized or minimized. This is done with the addition of external perturbation signal to the input signal of the plant, which generates variations in the output signal. The updated control signal is calculated on the basis of the change in the output signal.

There are known many algorithms for ESC but for some of them the stability is not mathematically proven. One of the frequently studied versions of this algorithm is the one using periodic external perturbation signal with sinusoidal form $[2,3,5]$. 
The perturbation signal has to be weak, but to generate enough change in the output. The form of the signal can be different including rectangular and even stochastic. The speed of convergence depends also on normalized power of the perturbation signal [10]. The plant must be stable, but it is allowed to change in time. The diagram of this algorithm is shown on figure 3 .

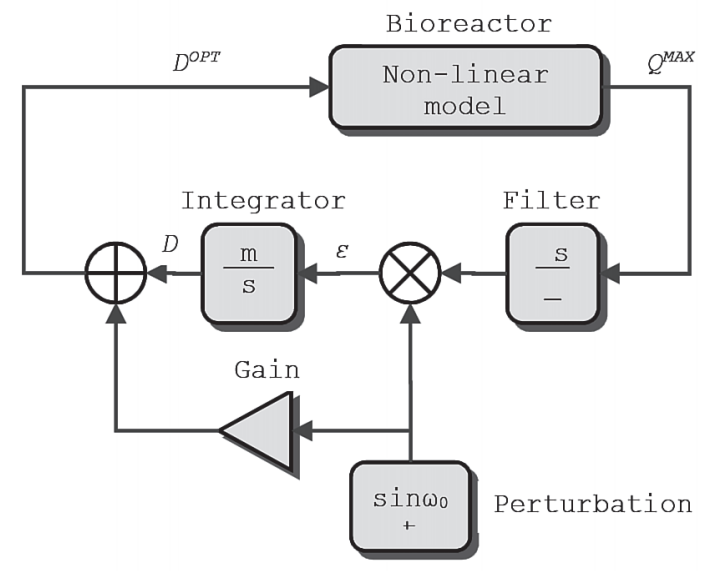

Figure 3. Diagram of sinusoidal ESC algorithm

Operating principle of the ESC control is the following. After suitable scaling of the external signal according to the plant, it is summed with the current control input D.

This signal generates periodic variations in output signal Q, which is passed through a high-pass filter eliminating the DC component. The filtered output signal is multiplied by the primary perturbation signal. After that the signal $\varepsilon$ is passed on to the integrator, which updates the control signal $\mathrm{D}$ in the direction where the gradient decreases. The signal $\mathrm{D}$ is also scaled with the coefficient $\mathrm{m}$.

A low-pass filter can be added before the integrator which passes only the DC component of the demodulated signal $\varepsilon$. In that case the calculated gradient will be exact, while if only integrator is used, the estimated gradient will not be completely accurate, because of the higher harmonics.

When the coefficient $m>0$, the algorithm is searching for maximum of the objective function and when $\mathrm{m}<0$ the minimum is searched. When $\varepsilon$ has positive value, the work point of the system is on the left of the maximum and vice versa. When the smooth function $\mathrm{Q}(\mathrm{D})$ has negative second derivative, it has a maximum and in the case of positive second derivative, it has minimum.

The parameters for tuning the ESC controller are coefficient $m$, the cut-off frequency of the high-pass filter $h$, the amplitude and a frequency $\omega 0$ of the periodic perturbation signal. The smaller the amplitude is, the smaller the residual error and output variations are, but the possibility for stopping at local extremum is higher and the speed of convergence is lower. Higher values of $\mathrm{m}$ lead to higher speed of convergence. The frequency $\mathrm{h}$ of the filter has to be sufficiently lower than that of the perturbation signal. The higher the $\omega 0$ is, the effect of the perturbation on the plant will be lower, but it has to stay in the frequency pass band of the plant.

\section{Cascade System Model}

The studied models are based on the mass balance of the process $[6,7]$. They are simplified and no inhibition nor microorganisms' death or any other features of $\mathrm{AD}$ process are taken into account.

By definition $\mathrm{D}=\mathrm{F} / \mathrm{V}$ and $\mathrm{F}=\mathrm{DV}$. From figure 2 is obvious that $\mathrm{F}=\mathrm{D}_{1} \mathrm{~V}_{1}=\mathrm{D}_{2} \mathrm{~V}_{2}$ or

(1) $V_{2}=\frac{D_{1}}{D_{2}} V_{1}=K V_{1}$.

This means that the working volume of $\mathrm{BR}_{2}$ is $\mathrm{K}$ times that of the $\mathrm{BR}_{1}$. It is known that the specific growth rate of methanogens is considerably lower than that of the hydrogen producing microorganisms, so $\mathrm{D}_{1}>\mathrm{D}_{2}$ and $\mathrm{K}>1$. After determining the optimum values $\mathrm{D}_{1}{ }^{2}{ }^{2}$ and $\mathrm{D}_{2}{ }^{\mathrm{OPT}}$ the value of $\mathrm{K}$ bringing $\mathrm{BR}_{2}$ to its optimal working point can be calculated [9].

In this paper the following two models of the first bioreactor $\left(\mathrm{BR}_{1}\right)$ are considered:

$$
\begin{aligned}
\frac{d S_{1}}{d t} & =-\frac{1}{Y_{1}} \mu_{1} X_{1}+D_{1}\left(S_{1}^{I N}-S_{1}\right) \\
\frac{d X_{1}}{d t} & =\mu_{1} X_{1}-D_{1} X_{1} \\
\text { (2) } \frac{d A c_{1}}{d t} & =\frac{1}{Y_{2}} \mu_{1} X_{1}-D_{1} A c_{1} \\
\mu_{1}= & \frac{\mu_{1}^{M A X} S_{1}}{K_{1}^{S}+S_{1}} \\
Q_{H 2} & =Y_{H 2} \mu_{1} X_{1}
\end{aligned}
$$

(3)

$$
\begin{aligned}
& \frac{d S_{0}}{d t}=-\beta X_{1} S_{0}+D_{1}\left(Y_{P} S_{0}^{I N}-\mathrm{S}_{0}\right) \\
& \frac{d S_{1}}{d t}=-D_{1} S_{1}+\beta X_{1} S_{0}-\frac{1}{Y_{1}} \mu_{1} X_{1} \\
& \frac{d X_{1}}{d t}=\mu_{1} X_{1}-D_{1} X_{1} \\
& \frac{d A c_{1}}{d t}=\frac{1}{Y_{2}} \mu_{1} X_{1}-D_{1} A c_{1} \\
& Q_{H 2}=Y_{H 2} \mu_{1} X_{1} \\
& \mu_{1}=\frac{\mu_{1}^{M A X} S_{1}}{K_{1}^{S}+S_{1}}
\end{aligned}
$$

The used notations are:

$\mathrm{S}_{0}, \mathrm{~S}_{1}$ - organics concentration in the $\mathrm{BR}_{1}[\mathrm{~g} / \mathrm{l}]$;

$\mathrm{S}_{0}{ }^{\mathrm{IN}}, \mathrm{S}_{1}{ }_{1}{ }^{\mathrm{IN}}-$ organic concentration in the inlet flow of $\mathrm{BR}_{1}[\mathrm{~g} / \mathrm{l}]$;

$\mathrm{X}_{1}$ - biomass concentration in the $\mathrm{BR}_{1}[\mathrm{~g} / \mathrm{l}]$;

$\mathrm{Ac}_{1}$ - acetate concentration in $\mathrm{BR}_{1}[\mathrm{~g} / \mathrm{l}]$;

$\mathrm{QH}_{2}$ - hydrogen flow rate per hour [dm3/l.h];

$\mu_{1}$ - specific growth rate of hydrogen producing microorganisms $\left[\mathrm{h}^{-1}\right]$. 
Model (2) describes a one-step transformation of organic substrate into hydrogen and acetate by one type of microorganisms. Model (3) adds hydrolysis as first step, which is conducted by the same microorganisms.

The bioreactors are operated in continuous mode where the transient processes are finished, so we can find the equations for the above variables in the steady-state, by nullifying the differential equations. After some other mathematical transformations we get the two systems of algebraic equations for both models which give the values as functions of model parameters and the two input signals, $\mathrm{D}_{1}$ and $\mathrm{S}^{\mathrm{IN}}$.

The input-output static characteristics of the bioreactors $\mathrm{Q}(\mathrm{D})$ are important for the extremum seeking control. The coordinates of their maximum are calculated by nullifying the first derivative of $Q(D)$ for several values of $\mathrm{S}^{\mathrm{IN}}$.

The model of the second bioreactor in the cascade $\left(\mathrm{BR}_{2}\right)$ is given in (4).

$$
\begin{aligned}
& \frac{d X_{2}}{d t}=\mu_{2} X_{2}-D_{2} X_{2} \\
& \frac{d A c_{2}}{d t}=-\frac{1}{Y_{3}} \mu_{2} X_{2}+D_{2}\left(A c_{1}-A c_{2}\right) \\
& \mu_{2}=\frac{\mu_{2}{ }^{M A X} A c_{2}}{K_{2}{ }^{s}+A c_{2}} \\
& Q_{C H 4}=Y_{C H 4} \mu_{2} X_{2}
\end{aligned}
$$$$
\text { (4) }
$$

It describes again a one-step transformation of the inlet acetate into methane by single type of microorganisms.

The used notations are:

$\mathrm{X}_{2}$ - biomass concentration in $\mathrm{BR}_{2}[\mathrm{~g} / \mathrm{l}]$;

$\mathrm{Ac}_{2}$ - acetate concentration in $\mathrm{BR}_{2}[\mathrm{~g} / \mathrm{l}]$;

$\mathrm{Ac}_{1}$ - inlet acetate from $\mathrm{BR}_{1}[\mathrm{~g} / \mathrm{l}]$;

$\mathrm{Q}_{\mathrm{CH} 4}-$ methane yield per hour $\left[\mathrm{dm}^{3} / 1 . \mathrm{h}\right]$;

$\mu_{2}$ - specific growth rate of methanogens $\left[\mathrm{h}^{-1}\right]$.

The corresponding equations for $\mathrm{D}_{1}{ }^{\mathrm{OPT}}$ and $\mathrm{D}_{2}{ }^{\mathrm{OPT}}$ are given in (5) and (6), respectably. The equations of $Q(D)$ for the models of $\mathrm{BR}_{1}$ and $\mathrm{BR}_{2}$ respectably, are given in (7) and (8). The expressions for $\mathrm{S}_{1}, \mathrm{Ac}_{1}$ and $\mathrm{Ac}_{2}$ in the steady-state mode are not given, but they can be expressed by model parameters and input signals in question. The value of $\mathrm{D}_{1}{ }^{\text {OPT }}$ for the second model of $\mathrm{BR}_{1}$ is numerically determined due to impossibility of Symbolic Math toolbox to find solution.

$$
D_{1}^{O P T}=\mu_{1}^{M A X}\left(1-\sqrt{\frac{K_{1}^{S}}{S_{1}^{I N}+K_{1}^{S}}}\right)
$$

(6)

$$
D_{2}{ }^{O P T}=\mu_{2}^{M A X}\left(1-\sqrt{\frac{K_{2}^{S}}{A c_{1}+K_{2}^{S}}}\right)
$$

(7) $Q_{H 2}=Y_{1} Y_{H 2} D_{1}\left(S_{1}^{I N}-S_{1}\right)$

(8) $Q_{\mathrm{CH} 4}=Y_{3} Y_{\mathrm{CH} 4} D_{2}\left(A c_{1}-A c_{2}\right)$

\section{Simulation Studies}

The computer simulations of the cascade system are done in Matlab/Simulink environment version 2009b and 2016a. The mathematical transformations are done with Symbolic Math Toolbox in the same products.

The models of both bioreactors are not calibrated due to the scarce experimental data. Because of that, example values for the model parameters are used, which leads to complex values in some of the simulations [11].

Simulations with two variants of the cascade system are made. The first variant uses first model of $\mathrm{BR}_{1}$ and the second variant uses the second, more complex, model of $\mathrm{BR}_{1}$. The model of $\mathrm{BR}_{2}$ is the same for the two variants of

\begin{tabular}{|c|c|c|c|c|c|c|}
\hline$Y_{1}$ & $\mathbf{Y}_{2}$ & $\mathbf{Y}_{\mathrm{H} 2}$ & $\begin{array}{c}\boldsymbol{\mu}_{1}{ }_{\mathbf{X}}^{\mathbf{M A}} \\
\end{array}$ & $\mathbf{K}_{1}{ }^{S}$ & $\boldsymbol{\beta}$ & $\mathbf{Y}_{\mathbf{P}}$ \\
\hline 0.08 & 1 & 1 & 0.568 & 3.914 & 1 & 1 \\
\hline$Y_{3}$ & $Y_{\mathrm{CH} 4}$ & $\boldsymbol{\mu}_{2}{ }^{\mathrm{MAX}}$ & $\mathbf{K}_{2} \mathbf{S}$ & & & \\
\hline 0.24 & 18.7 & 0.0083 & 0.22 & & & \\
\hline
\end{tabular}
the cascade system. In table 2 are given model parameters for both bioreactors and in table 3 the parameters of the ESC controller are given. They are chosen heuristically for the first model of $\mathrm{BR}_{1}$.

Table 2. Bioreactors model parameters

Table 3. ESC controller parameters

\begin{tabular}{|c|c|c|c|}
\hline $\begin{array}{c}\text { Amplitude } \\
\boldsymbol{a}\end{array}$ & $\begin{array}{c}\text { Frequency } \\
\boldsymbol{\omega}_{\boldsymbol{0}}\end{array}$ & $\begin{array}{c}\text { Frequency } \\
\boldsymbol{h}\end{array}$ & $\begin{array}{c}\text { Gain } \\
\boldsymbol{m}\end{array}$ \\
\hline $0.04 \mathrm{~h}^{-1}$ & $0.6 \mathrm{rad} / \mathrm{s}$ & $0.5 \mathrm{rad} / \mathrm{s}$ & 0.1 \\
\hline
\end{tabular}

\subsection{Volumetric Coefficient K}

The input-output static characteristics of the three models for two values of $\mathrm{S}^{\mathrm{IN}}$ are given on figures 4-6, respectably. In table 4 are given the calculated values of the important system parameters.

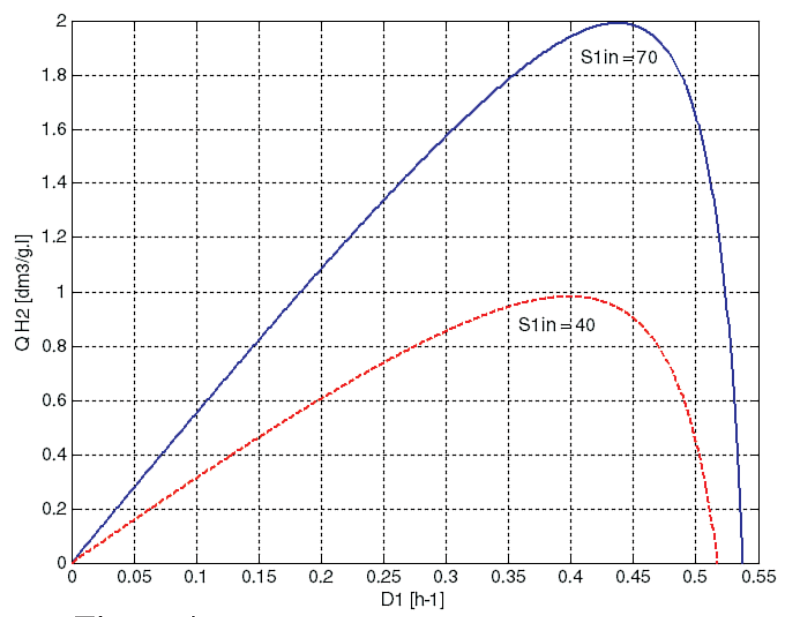

Figure 4. I/O static characteristics of BR1 model 1 


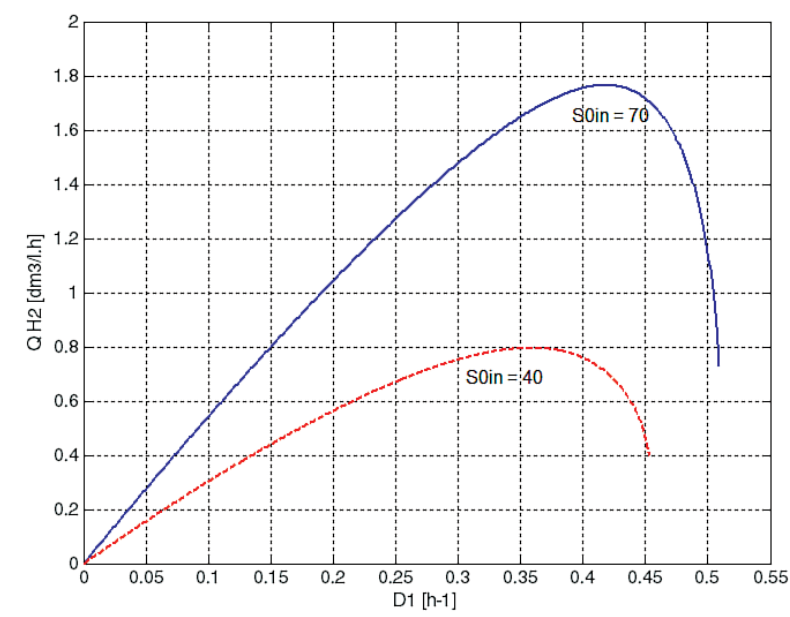

Figure 5. I/O static characteristics of $\mathrm{BR}_{1}$ model 2

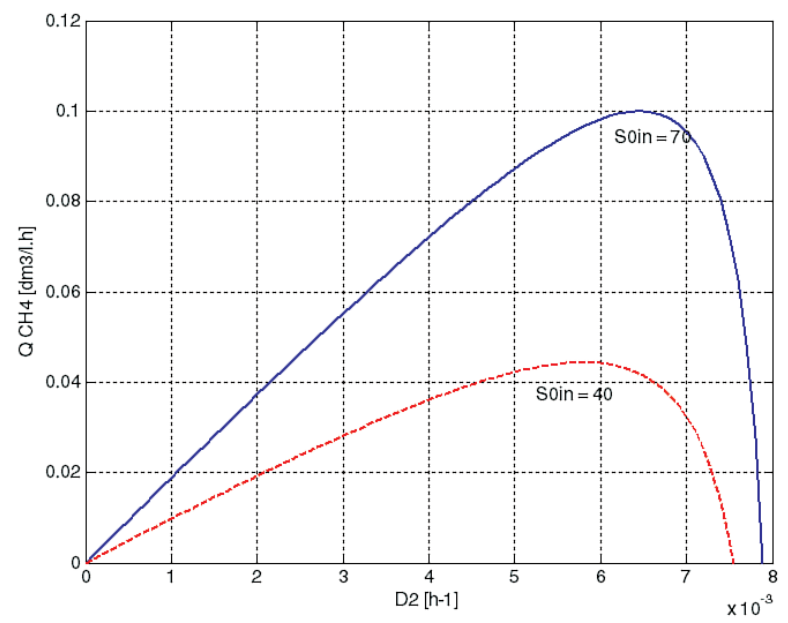

Figure 6. I/O static characteristics of BR2

Table 4. Values of $\mathrm{D}^{\mathrm{OPT}}, \mathrm{D}^{\mathrm{WASH}}$ and $\mathrm{Q}^{\mathrm{MAX}}$

\begin{tabular}{|c|c|c|c|c|c|c|}
\hline $\begin{array}{c}\mathrm{S}^{\mathrm{IN}} \\
{[\mathrm{g} / \mathrm{l}]}\end{array}$ & $\begin{array}{c}\mathrm{D}_{1}{ }^{\text {OPT }} \\
{\left[\mathrm{h}^{-1}\right]}\end{array}$ & $\begin{array}{c}\mathrm{D}_{1}{ }^{\text {WASH }} \\
{\left[\mathrm{h}^{-1}\right]}\end{array}$ & $\begin{array}{c}\mathrm{Q}_{1}{ }^{\mathrm{MAX}} \\
{\left[\mathrm{dm}^{3} / 1 . \mathrm{d}\right]}\end{array}$ & $\begin{array}{c}\mathrm{D}_{2}{ }^{\text {OPT }} \\
{\left[\mathrm{h}^{-1}\right]}\end{array}$ & $\begin{array}{c}\mathrm{D}_{2}{ }^{\text {WASH }} \\
{\left[\mathrm{h}^{-1}\right]}\end{array}$ & $\begin{array}{c}\mathrm{Q}_{2}{ }^{\mathrm{MAX}} \\
{\left[\mathrm{dm}^{3} / 1 . \mathrm{d}\right]}\end{array}$ \\
\hline 70 & 0.437 & 0.538 & 1.991 & 0.0065 & 0.0079 & 0.110 \\
\hline 40 & 0.398 & 0.517 & 0.986 & 0.0059 & 0.0076 & 0.051 \\
\hline \multicolumn{7}{|c|}{ Variant 2 } \\
\hline 70 & 0.418 & 0.510 & 1.770 & 0.0065 & 0.0079 & 0.100 \\
\hline 40 & 0.359 & 0.454 & 0.796 & 0.0058 & 0.0076 & 0.045 \\
\hline
\end{tabular}

From figures 4-6 it is obvious that the input-output characteristics have only global maximum and it is in the admissible range for control input $\mathrm{D}$. The coordinates of extremum is shifting down and left with lowering the organics concentration of the inlet flow $\mathrm{S}^{\mathrm{IN}}$. The slope after the maximum is considerably steeper and this can quickly render the process nonoperational, because further small increments of $\mathrm{D}$ lead to large decrease in $\mathrm{Q}$.

Making the model of $\mathrm{BR}_{1}$ a little more complex lead to lowering biogas flow rate, achieved at lower dilution rates also. The form of characteristics remained the same. The one on figure 5 is not complete, because the remaining part is complex, due to the model parameters.
From equations (5) and (6) for $\mathrm{D}^{\mathrm{OPT}}$ is obvious that they are functions of $\mathrm{S}^{\mathrm{IN}}$ and $A c_{1}$, but these variables are bound with the model of $\mathrm{BR}_{1}$. Therefore, the coefficient $\mathrm{K}$ will depend on the input signals $S^{I N}$ and $D_{1}$ of $B_{1}$. The characteristics $\mathrm{K}\left(\mathrm{S}^{\mathrm{IN}}, \mathrm{D}_{1}\right)$ for the two variants of cascade system and two values of $\mathrm{D}_{1}$ are given on figures 7 and 8 . The two values of $\mathrm{D}_{1}$ correspond to the optimum values for the two organic concentrations $\mathrm{S}^{\mathrm{IN}}$, before and after the disturbance in $\mathrm{S}^{\mathrm{IN}}$.

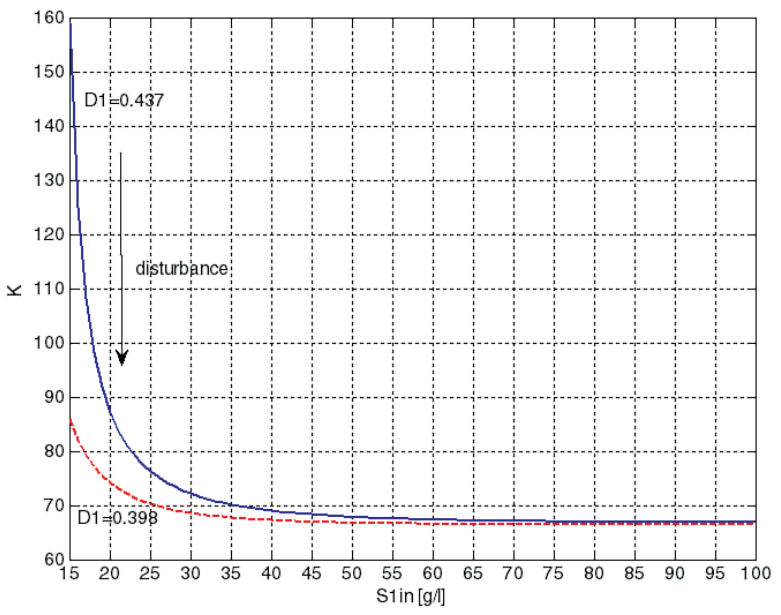

Figure 7. Characteristics $\mathrm{K}\left(\mathrm{S}^{\mathrm{IN}}, \mathrm{D}_{1}\right)$ for variant 1

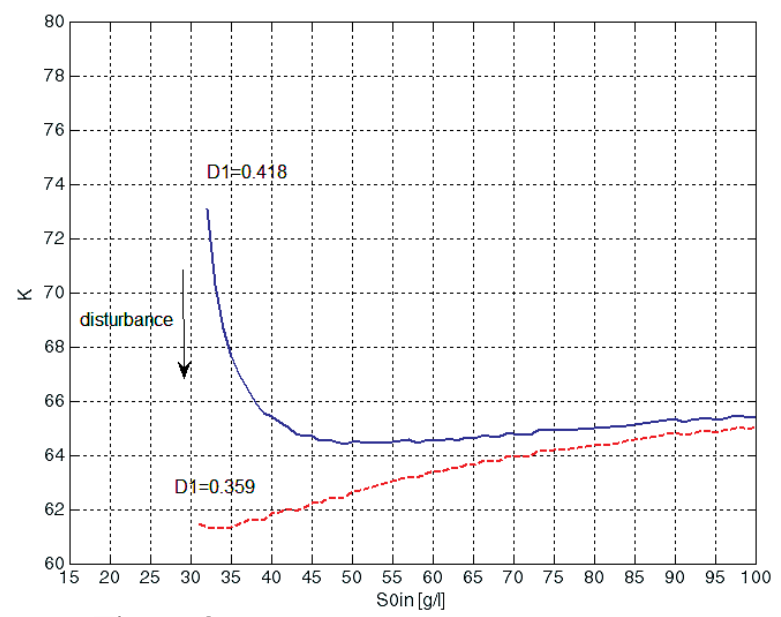

Figure 8. Characteristics $K\left(S^{I N}, D_{1}\right)$ for variant 2

As can be seen from figures 7 and 8 , K has very close values for high input concentrations, especially for variant 1 . The change in $\mathrm{K}$ for variant 2 is a little higher for the range of $\mathrm{S}^{\mathrm{IN}}=40-70 \mathrm{~g} / \mathrm{l}$. When the $\mathrm{S}^{\mathrm{IN}}$ changes, this leads to change in $\mathrm{D}_{1}{ }^{\mathrm{OPT}}$, so the optimum point shifts to the lower line in both figures. The calculated values of $\mathrm{K}$ before and after disturbance in $\mathrm{S}^{\mathrm{IN}}$ are given in table 5 .

Table 5. Values of $\mathrm{K}$ for the two variants and disturbance in $\mathrm{S}^{\mathrm{IN}}$

\begin{tabular}{|l|c|c|}
\hline & Before & After \\
\hline Variant 1 & 67.085 & 67.245 \\
\hline Variant 2 & 64.775 & 61.836 \\
\hline
\end{tabular}

For variant 1 , the change in $\mathrm{K}$ is $0.24 \%$ and there is no observed change is biogas flow rate $\mathrm{Q}_{\mathrm{CH} 4}$ before and after disturbance in the case of unchanged $K$. For variant 2, the 
change in $\mathrm{K}$ is $4.54 \%$ and the decrease in $\mathrm{Q}_{\mathrm{CH} 4}$ after disturbance is $1.54 \%$. Here, the working point remains on the left of the maximum of the $\mathrm{I} / \mathrm{O}$ static characteristic of $\mathrm{BR}_{2}$, where the decrease of biogas flow is slower.

When $\mathrm{K}$ is higher after disturbance, this means that the working point of $\mathrm{BR}_{2}$ remains on the right side of the maximum and vice versa, if no measures are taken to correct it. Therefore, the first situation is more problematic because $\mathrm{BR}_{2}$ gets closer to dangerous zone, where washout of microorganisms can occurs.

For the studied models of bioreactors the coefficient $\mathrm{K}$ remains almost the same for relatively large diapason of $\mathrm{S}^{\mathrm{IN}}$ and it is not mandatory to use additional controller to correct the working volume of the second bioreactor. The drop in biogas flow rate $\mathrm{Q}$ is very little if $\mathrm{K}$ keeps its first value, but if the disturbance in $\mathrm{S}^{\mathrm{IN}}$ is larger, $\mathrm{K}$ gets sufficiently different and this can causes the process in $\mathrm{BR}_{2}$ to stop or to function far from optimum working point.

\subsection{Extremum Seeking Control}

The objective function to be maximized in this paper is accepted as:

$$
A=Q_{H 2}+Q_{C H 4} \rightarrow \text { max. }
$$

As it was shown, if the little drop in $\mathrm{Q}_{\mathrm{CH} 4}$, due to the suboptimal value of $\mathrm{K}$, is neglected, the above function can be modified to include biogas flow of the first bioreactor only and this will be enough for optimum results of the cascade system for relatively large disturbances in $\mathrm{S}^{\mathrm{IN}}$ and the objective function obtain the form:

$$
A=Q_{H 2} \rightarrow \max
$$

The effect of ESC controller is tested for both variants of the cascade system, starting from left and right of the maximum. The starting values of $\mathrm{D}_{1}$ is 0 and the highest possible for which the process stays vital.

On figures 9 and 10 are given the trajectories of the working points of the two variants of system in the phase plane Q-D. Two simulations are performed starting with different $\mathrm{D}_{1}(0)$.

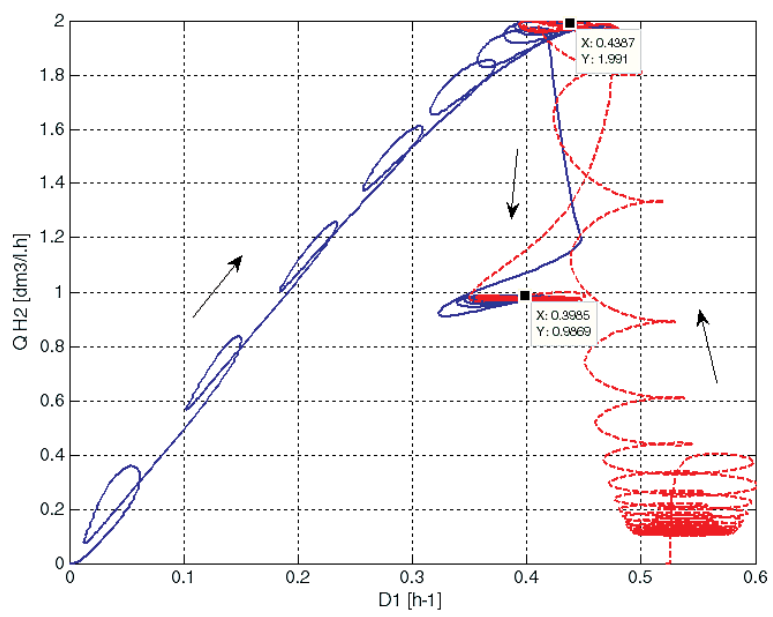

Figure 9. BR process with ESC control for variant 1 in phase plane, $\mathrm{D}^{\mathrm{RIGHT}}(0)=0.522$

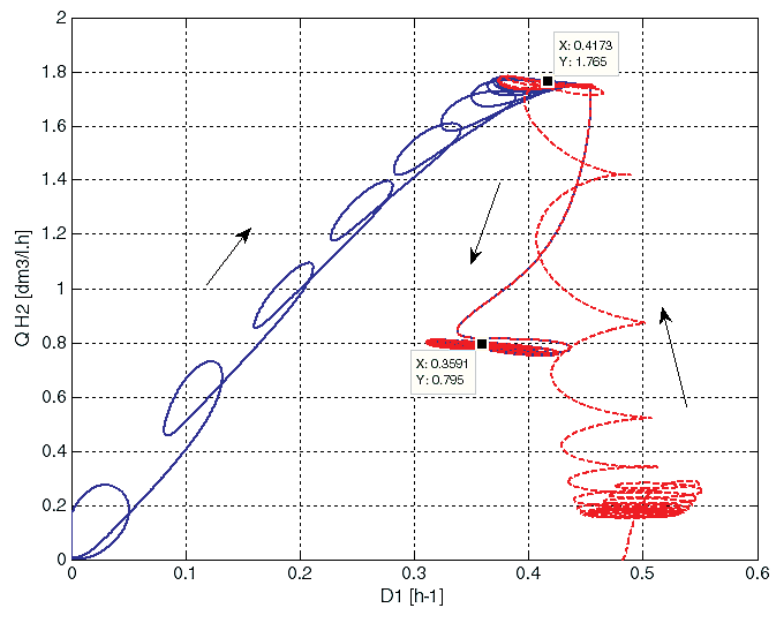

Figure 10. $\mathrm{BR}_{1}$ process with ESC control for variant 2 in phase plane, $\mathrm{D}^{\mathrm{RIGHT}}(0)=0.482$

From figures 9 and 10 it can be seen that even when the process start far from the optimum value in the danger zone, the ESC controller succeeds in finding the best value for $\mathrm{D}_{1}$ for both variants.

In table 6 are given some quality metrics of the cascade system for the two variants with ESC controller and the open loop system. The steady-state time of $\mathrm{H}_{2}$ is longer with ESC controller, partly because of the negative wave of the perturbation signal and partly of the controller parameters. They can be tuned, so the steady-state time is shortened. When the start is from the right the steady-state time is significantly longer for both biogases. Methane is getting to the steady-state faster for variant 2.

Table 6. Biogas flow with and without ESC

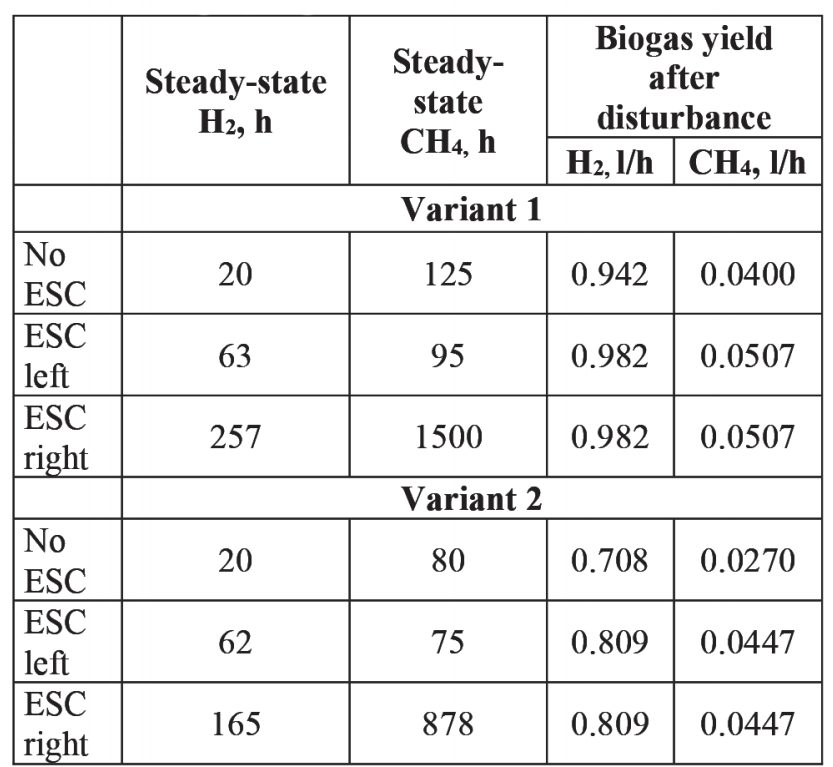

For variant 2, the difference in the flow rate of the methane $(39.6 \%)$ is caused by suboptimal coefficient $\mathrm{K}$ and dilution rate $\mathrm{D}_{1}$ when no ESC is used. For variant 1 , the difference is $21.1 \%$. The difference in the hydrogen flow rate is $4.1 \%$ for variant 1 and $12.5 \%$ for variant 2 . From these figures can be seen that the more complex model of $\mathrm{BR}_{1}$ 
leads to larger loss in the biogas yields, then the first model, when no ESC is used.

In table 7 are given the degree of biodegradation in the two bioreactors for both variants of the cascade system. The ESC control recovered the degree of biodegradation after the disturbance for both variants. The gain in the process efficiency when ESC is used is larger for variant 2. The negative impact of the disturbance is greater for the second bioreactor.

When using perturbation signal with amplitude of 0.04 $\mathrm{h}^{-1}(9.2 \%$ before and $10.1 \%$ after perturbation), periodic variations of hydrogen and methane are given in table 8. The perturbation is from 70 to $40 \mathrm{~g} / \mathrm{l}$ (step decrease of $43 \%$ ).

Table 7. Degree of biodegradation in both bioreactors in the cascade system

\begin{tabular}{|l|c|c|c|c|}
\hline \multirow{2}{*}{} & \multicolumn{2}{|c|}{ Before disturbance } & \multicolumn{2}{c|}{ After disturbance } \\
\cline { 2 - 5 } & $\mathbf{B R}_{\mathbf{1}}$ & $\mathbf{B R}_{\mathbf{2}}$ & $\mathbf{6} \mathbf{P}_{\mathbf{1}}$ & $\mathbf{6 P}_{\mathbf{2}}$ \\
\cline { 2 - 5 } $\begin{array}{l}\text { Variant } \mathbf{1 ,} \% \\
\text { ESC }\end{array}$ & 75 & 83 & 62 & 63 \\
\hline ESC & 75 & 83 & 70 & 78 \\
\hline & \multicolumn{5}{|c|}{ Variant $\mathbf{2 ,} \%$} \\
\hline $\begin{array}{l}\text { No } \\
\text { ESC }\end{array}$ & 70 & 82 & 49 & 55 \\
\hline ESC & 70 & 83 & 65 & 82 \\
\hline
\end{tabular}

Table 8. Periodic variations in the output of the bioreactors

\begin{tabular}{|c|c|c|c|c|}
\hline \multirow{2}{*}{} & \multicolumn{2}{|c|}{ BR $_{1}$ \% } & \multicolumn{2}{c|}{ BR $_{2}, \%$} \\
\cline { 2 - 5 } & Before & After & Before & After \\
\hline Variant 1 & 0.70 & 0.60 & 0.90 & 0.10 \\
\hline Variant 2 & 1.40 & 2.10 & 0.08 & 0.07 \\
\hline
\end{tabular}

The ESC behavior is studied also in the case of varied parameters of model 2 of the $\mathrm{BR}_{1}$. The two examined coefficients are connected with the hydrolysis step, $\beta$ and $\mathrm{Y}_{\mathrm{P}}$. Their values are given in table 9. The results from simulations are presented on figure 11.

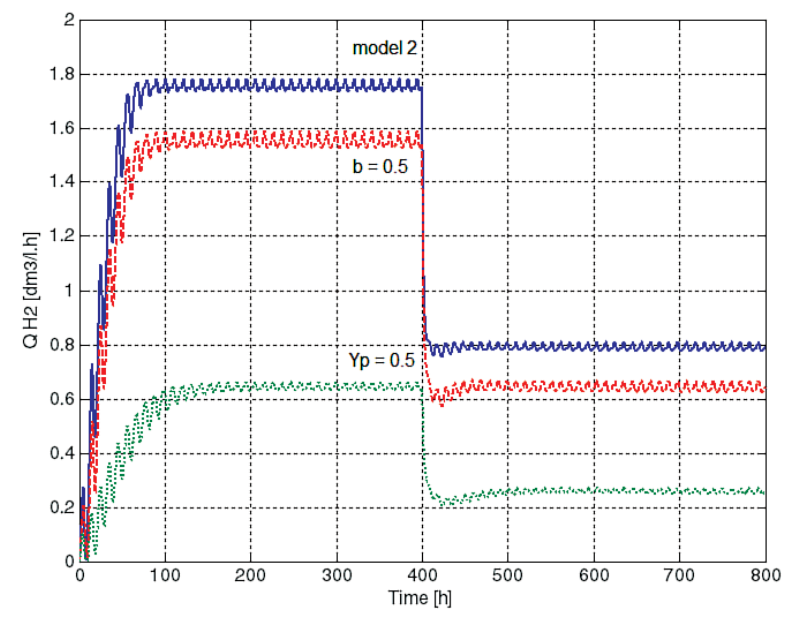

Figure 11. ESC control of BR1 and varied model parameters in the time domain
Table 9. Values of $\mathrm{Q}_{1}^{\mathrm{MAX}}$ and $\mathrm{D}_{1}^{\mathrm{OPT}}$ before disturbance for model 2 and varied parameters

\begin{tabular}{|l|c|c|}
\hline Coefficient & $\begin{array}{c}\mathbf{Q}_{\mathbf{1}}{ }^{\text {MAX }}, \\
\mathbf{d m}^{\mathbf{3}} / \mathbf{l} . \mathbf{h}\end{array}$ & $\mathbf{D}_{\mathbf{1}}{ }^{\text {OPT }} \mathbf{~ h}^{\mathbf{1}}$ \\
\hline Model 2 & 1.775 & 0.418 \\
\hline$Y_{\mathbf{P}}=0.5$ & 0.659 & 0.341 \\
\hline$\beta=0.5$ & 1.571 & 0.396 \\
\hline
\end{tabular}

It is obvious that these changes in parameters have effect mainly on the biogas flow rate and not on the dynamics of the bioreactor. So, the previously configured for the first model of BR1, ESC controller gives very good results even for those models. The influence of YP is much greater than that of $\beta$, for both, biogas flow rate and dilution rate. The ESC control succeeds in finding the optimal control action for these models too. This means that the ESC control has the properties of a robust control system

\section{Conclusion}

In the paper was discussed the problem of improving biogas energy production of $\mathrm{AD}$ processes in a cascade of continuously stirred tank bioreactors with hydrogen and methane production. For this task simple mathematical models of bioreactors were adopted. It was shown that the ESC control strategy can be suitable solution for this task and that for the studied bioreactor models it is not mandatory to use another controller for the inner loop of the cascade system correcting the working volume of the second bioreactor in order to obtain close to the maximum system performance.

The ESC found optimal dilution rate of the substrate when started from 0 and close to the washout value of $D_{1}$. The algorithm updated the control signal to the new optimal value in the presence of disturbance in the inlet organics concentration $\mathrm{S}^{\mathrm{IN}}$.

The ESC controller was tuned for the first model of $\mathrm{BR}_{1}$, but it achieved good performance without being retuned for the second model too and even with changed model parameters. This shows that the ESC approach is viable solution for bioreactors control where many variables are hard to measure and that it has robust properties.

All studied in this paper bioreactor models are simplified and they do not account for many real-life effect such as inhibition by substrate, existing of more microorganisms groups and alternative pathways for substrate transformation. These features will have significant effects on the ESC control system performance and optimal volumetric coefficient determination.

The existing research papers have shown that this algorithm can be further improved by different modifications and to acquire even better results [13].

\section{Acknowledgement}

The authors gratefully acknowledge the financial support of this work by the Bulgarian National Science Fund, contract No. DFNI-E02/13. 


\section{References}

1. Deublein, D. and A. Steinhauser. Biogas from Waste and Renewable Resources. Wiley-VCH Verlag, Weinheim, 2008.

2. Krstic, M., H.-H. Wang. Stability of Extremum Seeking Feedback for General Nonlinear Dynamic Systems. - Automatica, 36, 2000, 595-601.

3. Dochain, D. (Ed.) Automatic Control of Bioprocesses, Chapter 7. Adaptive Linearizing Control and Extremum-seeking Control of Bioprocesses. John Wiley and Sons, 2008, 242.

4. Harmand, J., D. Dochain. The Optimal Design of Two Interconnected (Bio)Chemical Reactors Revisited. - Computers \& Chemical Engineering, 30, 2005, 70-82.

5. Simeonov, I., N. Noykova and M. Gyllenberg. Identification and Extremum Seeking Control of the Anaerobic Digestion of Organic Wastes. - Cybernetics and Information Technologies, 7, 2007, 2, 73-84.

6. Simeonov, I. Chapter 2. Dynamical Modeling and Estimation in Wasterwater Treatement Process. Contemporary Approaches to Modeling, Optimisation and Control of Biotechnological Processes. Tzonkov, S. (Ed.), Sofia, Prof. M. Drinov Acad. Publishing House, 2010.

7. Guo, X. M. et al. Hydrogen Production from Agricultural Waste by Dark Fermentation: a Review. - Int. J. Hydrogen Energy, 36, 2010, 10660-73.

\section{Manuscript received on 17.02.2017}

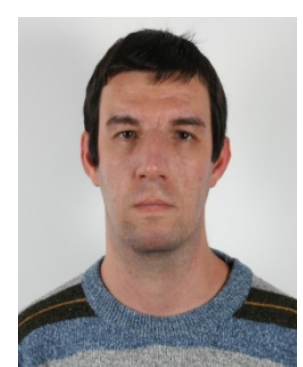

Eng. Vasil Lakov received his BSc and MSc degrees in Automation, Information and Control Systems from Technical University of Sofia in 2006. Currently, he is working at Stephan Angeloff Institute of Microbiology, Departament of Applied Microbiology. His research interests are in the fields of process automation, computer vision systems and image processing algorithms. He has a total of 10 published papers in the fields of microbiology, computer networks and road traffic control.

Contacts:

"Stephan Angeloff" Institute of Microbiology, Bulgarian Academy of Sciences 26 Acad. Georgi Bonchev St., 1113 Sofia, Bulgaria E-mail:vasil.lakov@yahoo.com

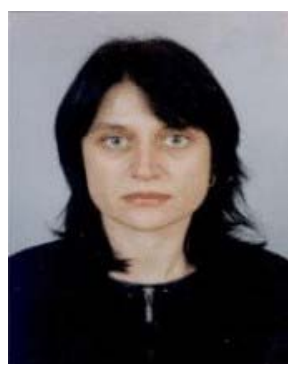

Res. Assoc. Elena Chorukova, PhD received her MSc degree in Bioengineering from the Technical University of Sofia, Bulgaria in 1994. Since 2000 she has been a Research Associate at the Bulgarian Academy of Sciences, in the research group "Mathematical Modeling and Computer Sciences" of the Stephan Angeloff Institute of Microbiology. She received her PhD degree in Bioengineering from the same institute in 2009.
8. Pakarinen, O. M. et al. Hydrogen and Methane Yields of Untreated, Water-extracted and Acid $(\mathrm{HCl})$ Treated Maize in One- and Two-stage Batch Assays. - Int. J. Hydrogen Energy, 36, 2011, 14401-144407.

9. Simeonov, Ivan, Elena Chorukova. Mathematical Modeling of the Anaerobic Digestion with Production of Hydrogen and Methane. The 4th International Conference on Water, Energy and Environment (ICWEE), Burgas, 1-2 June 2016, 32-38.

10. Nešić, Dragan. Extremum Seeking Control: Convergence Analysis. - European Journal of Control, 2009, 331-347.

11. Borisov, Milen, Neli Dimitrova, Ivan Simeonov. Mathematical Modelling of Anaerobic Digestion with Hydrogen and Methane Production. 6th IFAC Conference on Foundations of Systems Biology in Engineering, Magdeburg, Germany, 9-12 October 2016. 12. Gaida, Daniel, Christian Wolf, Michael Bongards. Feed Control of Anaerobic Digestion Processes for Renewable Energy Production: A Review. - Renewable \& Sustainable Energy Reviews, 68, 2017, Part 2, 869-875.

13. Liu, S.-J., M. Krstic. Stochastic Averaging and Stochastic Extremum Seeking. Communications and Control Engineering, Springer-Verlag London, 2012, Chapter 2.

She is an author/co-author of about 30 scientific papers published in international journals and conference proceedings.

$$
\begin{array}{r}
\text { Contacts: } \\
\text { "Stephan Angeloff" Institute of Microbiology, } \\
\text { Bulgarian Academy of Sciences } \\
26 \text { Acad. Georgi Bonchev St., 1113 Sofia, Bulgaria } \\
\text { E-mail: elena@microbio.bas.bg }
\end{array}
$$

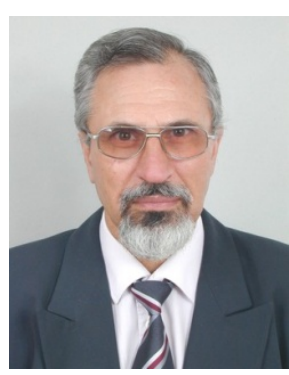

Assoc. Prof. Ivan Simeonov graduated from the Technical University of Sofia (1971) and specialized in l'Ecole Superieur d'Electricite and Laboratoire de Genie Electrique de Paris, France (1971-1973). He received his $\mathrm{PhD}$ degree from the same university in 1981. Since 1981 he has worked in the Bulgarian Academy of Sciences (BAS). The research achievements of Ivan Simeonov are published in more than 240 scientific articles and five monographs with more than 350 citations. At present Ivan Simeonov is the head of a research group at the Stephan Angeloff Institute of Microbiology, BAS

Contacts:

"Stephan Angeloff" Institute of Microbiology Bulgarian Academy of Sciences 26 Acad. Georgi Bonchev St., 1113 Sofia, Bulgaria E-mail: issim@microbio.bas.bg 